\title{
Im Pflanzenporträt: Die Süßholzwurzel (Glycyrrhiza radix)
}

Sabine Israel

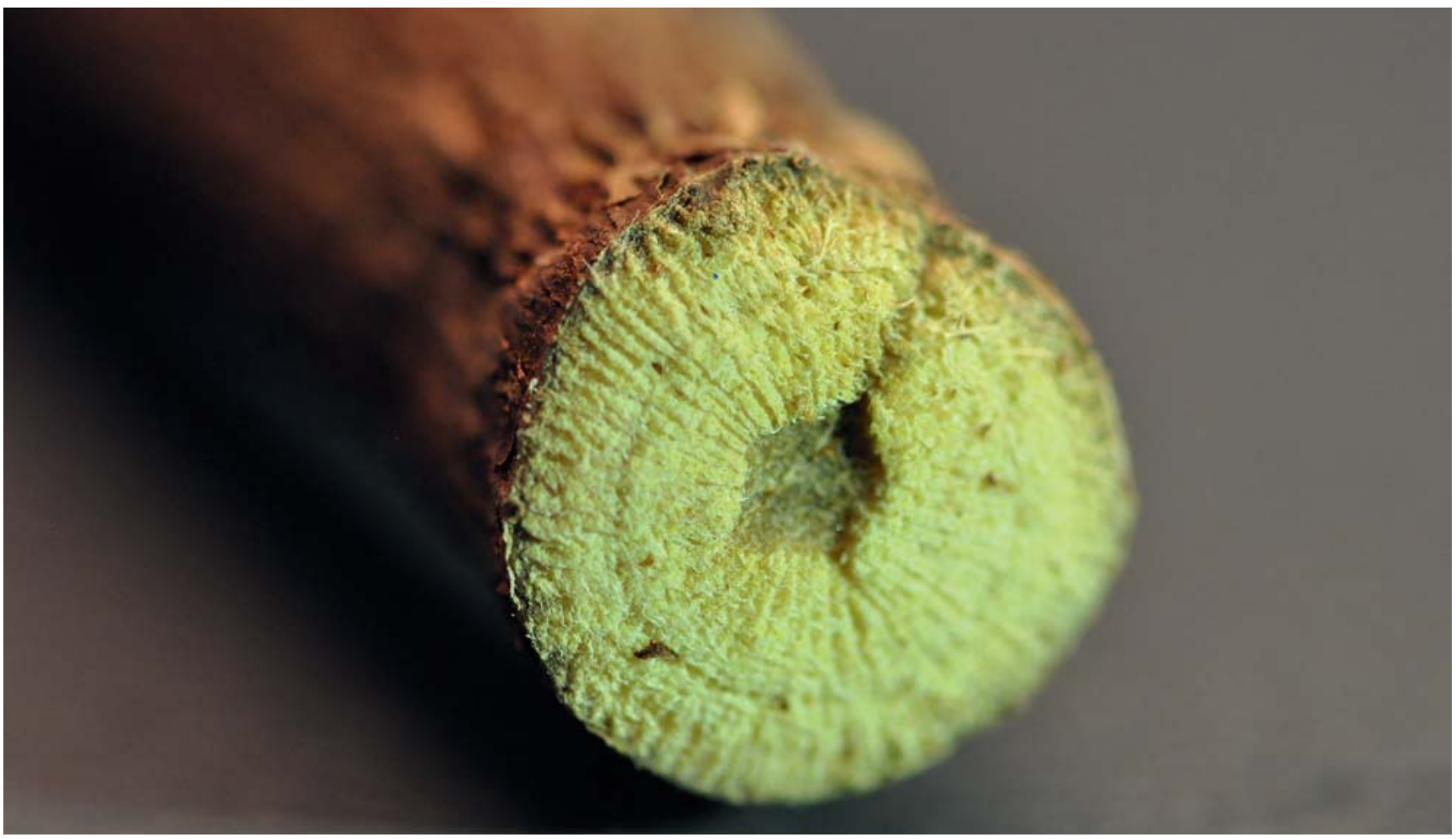

Die Süßholzwurzel enthält über 400 Inhaltsstoffe, die sie zu einer therapeutisch interessanten und in zahlreichen Studien getesteten Droge machen. Quelle: Sabine Israel

Seit dem 14. Jahrhundert ist der eingedickte Saft der Süßholzwurzel in Deutschland als Lakritze bekannt. Glycyrrhiza radix wird jedoch schon seit Jahrtausenden in der Volksmedizin geschätzt. Was sind ihre wichtigsten Inhaltsstoffe und wie wirken diese sich auf Mensch und Tier aus? Dass das Süßholz vielfältige positive Einflüsse auf den Körper hat und weit mehr ist als nur eine Süßigkeit oder ein Geschmackskorrigens für Arzneien, beweisen zahlreiche folgend angesprochene wissenschaftliche Studien u. a. auch zu SARS und COVID-19.

\section{Geschichte des Süßholzes}

Erstmals schriftlich erwähnt wurde Süßholz in einer mesopotamischen Schrift 1200 v. Chr. Die medizinische Anwendung bei Husten mit Auswurf, Magenkrankheit, Gelbsucht und Juckreiz beschreibt die Drogenliste Sultan Tepes aus Nordsyrien im 8. Jh. v. Chr.
Die Skythen, Reiternomaden der eurasischen Steppe, brachten das Süßholz nach Griechenland, wo die süße Wurzel (übersetzt: Glycyrrhiza) ihren heutigen Namen erhielt. Theophrast beschrieb die wasserretinierende und durstlöschende Wirkung des Süßholzes, die den Nomaden lange Wüstendurchquerungen ermöglichte. Griechen und Römer verwendeten Süßholz neben den bereits genannten Erkrankungen auch in Rezepturen gegen Nierenleiden und Blasenentzündung sowie bei Fußschmer- 
zen. Äußerlich wurde Süßholzwurzel als Augensalbe angewendet, bei Ischias- und Hüftschmerzen aufgetragen sowie fein pulverisiert in Honig auf Wunden aufgebracht. Ein Mundwasser aus Süßholz und Maulbeersaft half bei Aphten. Glycyrrhiza galt als allgemeines Stärkungsmittel und als Antidot bei unbekannten Giften.

Im antiken China gehörte Süßholz zu den 120 Drogen 1. Klasse, die das menschliche Leben erhalten und dem Himmel ähneln. Diese Heilpflanzen galten als ungiftig, den Körperzustand verbessernd und als geeignete Helfer, alt zu werden, ohne zu altern [1]. Konstantin von Afrika (1060 n. Chr.), ein weitgereister, arabisch-griechisch geprägter Mediziner an der Schule von Salerno, beschreibt die universelle Stärkung durch Süßholz: „Denn Luft ist Geist und Atmung geistige Tätigkeit, daher ist die Stärkung von Lunge und Herz auch eine Stärkung des Geistes." [2]

Deutschland erreichte die Süßholzpflanze der Sage nach durch die Heilige Kunigunde, die sie um 1000 n. Chr. als Gemahlin von Kaiser Heinrich II ihrer Lieblingsstadt Bamberg schenkte. Das Bamberger Umland entwickelte sich daraufhin zu einem bedeutenden Anbaugebiet und wurde bekannt für seine aus eingedicktem Süßholzsaft hergestellten Leckeritze (1394) bzw. später Lakritze (1429) und Süßholzküchle. Hildegard von Bingen notierte im 12. Jh., als die Süßholzwurzel bereits fester Bestandteil der Kloster- und Kräutermedizin war: „Es [das Süßholz] ist gut für die klare Stimme, helle Augen und einen milden Sinn. Auch dem Geisteskranken hilft es, weil es die Wut, die in seinem Gehirn ist, auslöscht." [2]

Heute liegen die Hauptanbaugebiete des Süßholzes in China, Russland, der Türkei, Italien, Bulgarien und Spanien [3]. Neben der arzneilichen Nutzung ist es häufiger Aromabestandteil in Nahrungsmitteln, Süßigkeiten, Getränken und amerikanischem Tabak. Nach Entzug der arzneilich relevanten Inhaltsstoffe können die ligninreichen Wurzeln zu Isolationsmaterialien weiterverarbeitet werden. Die schäumenden Eigenschaften der Süßholzwurzel werden bei Getränken und Feuerlöschern genutzt [2].

\section{Pflanzenbeschreibung: G. glabra}

Die Gattung der Süßhölzer (Glycyrrhiza) gehört zur Familie der Schmetterlingsblütler (Fabaceae) und umfasst ca. 30 verschiedene Arten. Drei davon werden medizinisch genutzt [3]:

- G. glabra L. (russisches oder spanisches Süßholz; in der deutschsprachigen Literatur oft auch als Echtes Süßholz bezeichnet. z.B. in [14])

- G. inflata BAT (aufgeblasenes oder chinesisches Süßholz)

- G. uralensis Fisch

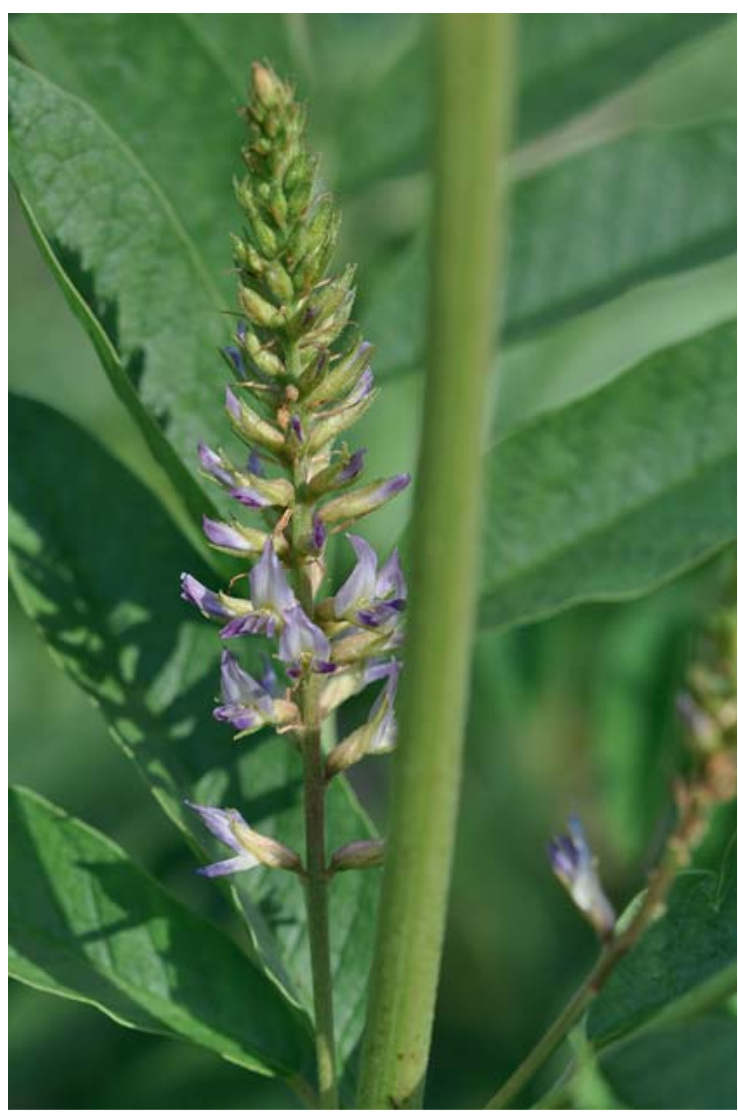

- Abb. 1 Im Frühsommer erkennt man das Echte Süßholz an seinen zarten, lilafarbenen Schmetterlingsblüten. Quelle: Sabine Israel

G. glabra ist heimisch im Mittelmeergebiet, Mittel- und Südrussland und Kleinasien bis Persien. G. uralensis und G. inflata sind in Ostasien, China und Russland beheimatet. Folgend wird das in unseren Breiten vorkommende G. glabra beschrieben.

Als mehrjährige, frosttolerante, krautige Pflanze erreicht Süßholz eine Wuchshöhe von 50-150 cm. Eine dicke, holzige Pfahlwurzel bildet zahlreiche Nebenwurzeln und meterlange Ausläufer. Die verholzten, faserigen Wurzeln sind gelb gefärbt mit graubrauner Rinde. Im oberirdischen Teil entspringen verzweigte, drüsig punktierte, stachelige Stängel, an denen wechselständige, kurz gestielte, bis $20 \mathrm{~cm}$ lange Blätter sitzen. Sie bestehen aus 9-17 elliptisch abgerundeten, bis $5 \mathrm{~cm}$ langen und $2 \mathrm{~cm}$ breiten Fiederblättern, an deren Unterseite harzig klebrige Drüsen sitzen. Aus den Blattachseln wachsen im Juni langstielige, traubenförmige Blütenstände mit ca. $1 \mathrm{~cm}$ großen, lilafarbenen Schmetterlingsblüten ( $\triangleright$ Abb. 1). Die glockenförmig verwachsenen 5 Kelchblätter bilden spitze Kelchzähne. Bei den 5 Blütenkronblättern sind die Flügelblätter kürzer als die Schiffchen, welche nicht verwachsen und nicht geschnäbelt sind. Die zur Röhre verwachsenen Staubfäden umschließen den Fruchtknoten. 
- Tab. 1 Dosierungsangaben für die innerliche Anwendung von Süßholzwurzel [14].

\begin{tabular}{|l|l|}
\hline Tierart & Tagesdosis \\
\hline Pferd & $10-60 \mathrm{~g}$ \\
\hline Rind & $25-100 \mathrm{~g}$ \\
\hline Schaf, Ziege & $5-15 \mathrm{~g}$ \\
\hline Lamm & $0,2-1,2 \mathrm{~g}$ \\
\hline Schwein & $5-10 \mathrm{~g}$ \\
\hline Ferkel & $0,1-1,0 \mathrm{~g}$ \\
\hline Hund & $0,1-2,0 \mathrm{~g}$ \\
\hline Katze* & $0,05-1,0 \mathrm{~g}$ \\
\hline Huhn & $0,1-1,0 \mathrm{~g}$ \\
\hline * Vorsicht: Süßholzwurzel ist für Katzen nur eingeschränkt \\
$\begin{array}{l}\text { geeignet; wiederholte Anwendung nur nach strenger Indika- } \\
\text { tion und maximal jeden 2. Tag. }\end{array}$ \\
\hline
\end{tabular}

Aus einem Fruchtblatt entwickelt sich die oberständige, bis $3,5 \mathrm{~cm}$ lange und $0,5 \mathrm{~cm}$ breite, gelegentlich leicht behaarte, rotbraune, gerade Hülse, die bei Vollreife 2-5 braune, $3 \mathrm{~mm}$ große, abgeflachte Samen entlässt. Bei der Ernte werden die oft meterlangen, dreijährigen Wurzeln so geschnitten, dass die Stammpflanze erhalten bleibt.

\section{Die Süßholzwurzel als Droge}

Zugelassen sind seit 2005 die getrockneten Wurzeln der 3 zuvor genannten, medizinisch genutzten Arten. Sie stammen ausschließlich aus kultiviertem Anbau [3].

Die Süßholzwurzel enthält über 400 Inhaltsstoffe. Die pharmakologisch wichtigsten sind:

- 2-15\% Triterpensaponine, wie Glycyrrhizin (50 × süßer als Rohrzucker) und 18ß-Glycyrrhetinsäure

- $10 \%$ Polysaccharide

- 0,65-2\% Flavonoide, z. B. das Chalcon Isoliquiritigenin, Isoliquiritin (gelbe Wurzelfarbe), Licochalcon A und prenylierte Isoflavone, z. B. Glabrin

- 0,04-0,06\% ätherisches Öl, Cumarine und Phytoöstrogene $[3,4]$

Dosierungsangaben für die innerliche Anwendung von Süßholzwurzel bei Tieren sind in ₹ Tab. 1 aufgelistet.

\section{ZUSATZINFO}

\section{Namenserklärung}

Süßholzwurzel $=$ Liquiritiae radix

Süßholzsaft = Liquiritiae succus

\section{Volksmedizinische Nutzung der Süßhölzer}

Süßholz entfaltet seine Kräfte vor allem dann, wenn der Körper ein wenig geschwächt ist: z. B. in einer Umbruchphase, wie der Menopause, in der Rekonvaleszenz oder unter psychischem Druck. Es vermag Entzündungen im ganzen Körper zu lindern, wobei der Schwerpunkt im Atmungs- und Verdauungstrakt liegt.

Zahlreiche Husten- und Bronchialtees sowie Lutschpastillen nutzen die sekretolytischen, auswurffördernden und bei Halsschmerzen entzündungshemmenden Eigenschaften des Süßholzes. Es schützt die Magenschleimhaut und wirkt krampflösend bei nervösen oder chronischen Gastritiden sowie Obstipationen im Verdauungstrakt [5]. Das Kauen der Wurzelstücke soll übrigens nicht nur bei Magenleiden, sondern auch bei einem Kater hilfreich sein. Weitere volksheilkundliche Anwendung findet Süßholz bei Entzündungen des Urogenitaltrakts, Rheumatismus, Arthritis, Hautkrankheiten wie Psoriasis, Epilepsie, zur Gedächtnisstärkung, bei Sexualschwäche und zur Förderung der Milchbildung [4].

Ethnoveterinärmedizinische Studien fanden bei Tieren eine Nutzung von Süßholz bei Erkrankungen des MagenDarm-Trakts, bei Atemwegserkrankungen sowie als Antiparasitikum und Repellent [6].

Die HMPC erachtet die traditionelle Anwendung bei dyspeptischen Beschwerden, Sodbrennen und als Expectorans bei erkältungsbedingtem Husten als sinnvoll, die ESCOP die unterstützende Therapie bei Magen- und Zwölffingerdarmgeschwüren sowie bei Gastritis und als Expectorans bei Husten und Bronchialkatarrh.

In China zählt die Süßholzwurzel zu den wichtigsten tonisierenden Heilpflanzen und findet sich in mehr als der Hälfte der klassischen Rezepturen. Im amtlichen chinesischen Arzneibuch von 1985 steht zur Süßholzwurzel: roh zur allgemeinen Kräftigung der Mitte (Milz/Magen) und geröstet zur Anhebung des Qi [1].

Die TCM unterscheidet 2 Zubereitungsarten: die getrocknete Wurzel Gan Cao und die in Honig geröstete Süßholzwurzel Zhi Gan Cao. Gan Cao hat ein neutrales Temperaturverhalten, klärt Hitze und vermag durch seine entgiftenden Eigenschaften die toxischen Wirkungen stärkerer Arzneien in der Rezeptur zu mildern und harmonisieren. Zhi Gan Cao ist warm und stärker tonisierend, aber ohne entgiftende Wirkung. Beide Formen haben einen Bezug zu allen Funktionskreisen, stärken aber speziell Herz, Lunge, Milz und Magen. In Kombination mit anderen Pflanzen finden sie sich in Rezepturen zur Befeuchtung der Lunge bei trockenem Husten oder Bluthusten, Dyspnoe und Auszehrung, spontanem oder nächtlichem 
Schwitzen, Hals- und Zungentrockenheit, Obstipation und im Falle von chronischem Fieber zur Stärkung des Lungen Qi und zur Behebung von Yin Mangel. Gan Cao wird speziell bei Rachen- und Halsschmerzen sowie Heiserkeit eingesetzt, wohingegen Zhi Gan Cao durch seine stärker tonisierende Wirkung zur Stärkung des Herz Qi bei Palpitationen, Herzrhythmusstörungen, Reizbarkeit, Schlafstörungen und Kurzatmigkeit, aber auch zur Abschwächung von Spasmen und zur Linderung von Schmerzen in Sehnen und Muskeln dient [1].

\section{Süßhölzer in pharmakologischen Studien}

Folgend werden die grundlegenden therapeutischen Eigenschaften der Süßholzwurzel, ihre Wirkung auf die jeweiligen Körperareale sowie die Ergebnisse dazugehöriger pharmakologischer Studien beschrieben.

\section{Grundlegende therapeutische Eigenschaften Antioxidative Wirkung}

G. glabra besitzt aufgrund der enthaltenen Flavonoide und Polysaccharide [4] gute antioxidative Eigenschaften. Studien konnten für verschiedene Organsysteme, wie Haut, Gehirn, Atmungstrakt, Herz und Blutgefäße, Leber und Niere, die protektive Wirkung gegen oxidativen Stress belegen [4,7].

\section{Antiinflammatorische Wirkung}

Die im Süßholz enthaltene Glycyrrhetinsäure verlangsamt durch Hemmung zweier Enzyme den Abbau körpereigenen Cortisols und wirkt somit entzündungshemmend und antiallergisch. Zusätzlich beeinflussen Glabridin und Isoliquiritin über Hemmung der Lipoxygenase und der Cyclooxygenase (Glabridin) sowie von Prostaglandin E2, Leukotrienen und Thromboxan (Isoliquiritin) das Entzündungsgeschehen [4].

\section{Immunmodulierende Wirkung}

In Fütterungsversuchen bei Schweinen [7] und Hühnern, die 0,1 \% Süßholzextrakt zum täglichen Futter bekamen, verbesserte sich die Immunreaktion auf spezifische und unspezifische Antigene deutlich und es konnte ein Anstieg der weißen Blutkörperchen im Blut beobachtet werden [8]. Synergie mit einer Impfung gegen virale Entenhepatitis wurde über Aktivierung der T-Lymphozyten beobachtet [7].

\section{Antibakterielle Wirkung}

Im Rahmen aktueller Resistenzforschung zeigten die Süßholzflavonoide eine gute bakteriostatische Wirkung gegen grampositive und gramnegative Erreger, wie Streptococcus mutans, Staphylococcus aureus, MRSA, Escherichia coli, Bacillus subtilis und Mycobakterium tuberculosis. Als sog. „resistance modifier“ kann G. glabra resistente Keime für bestimmte Antibiotika wieder sensibilisieren. Außerdem kann Süßholzwurzelextrakt die Bildung eines Biofilms und pathogener Exotoxine bei Sta- phylococcus aureus hemmen - beides wichtige Eigenschaften zur Behandlung von Wundinfektionen [7, 9]. In einem Mäuseversuch zeigte $G$. glabra Wirksamkeit gegen einen multiresistenten Pseudomonas aeruginosa und reduzierte die bakterielle Last im Blut.

\section{Antiparasitäre Wirkung}

Chalcone, wie Licochalcon A aus G. inflata, greifen in die Atmungskette von Parasitenmitochondrien ein. So konnte bei Mäusen Plasmodium yoelli vollständig eliminiert werden. Toxische Nebenwirkungen traten nicht auf. Invitro-Versuche mit Plasmodium falciparum, Leishmania donovani, Babesien und Theilerien bestätigen die Wirksamkeit der Süßholz-Chalcone gegen Plasmodien [10].

\section{Antivirale Wirkung}

Neben seiner allgemeinen Immunstärkung besitzt Süßholz antivirales Potenzial. In Versuchen mit dem Newcastle Virus bei Hühnerembryonen und dem Paramyxovirus Typ 1 bei Tauben vermochte Süßholzextrakt das Virus in Schach zu halten [8].

Mäuse, die intraperitoneal $10 \mu \mathrm{g}$ Glycyrrhizin/kg einen Tag vor und am Tag 1 und 4 nach einer letalen Influenza-A-Virusdosis bekamen, überlebten alle. Die Kontrollgruppe verstarb vollständig [11]. In Versuchen mit einigen DNA- und RNA-Viren zeigte sich, dass Glycyrrhetinsäure die Virusanheftung und die Virusvermehrung hemmt und zeitgleich die Produktion von Interferon y stimuliert. Dasselbe geschah in Zellkulturen mit SARS-CoVErregern aus klinischen Isolaten von im Jahre 2003 Erkrankten. Somit rückten Süßholz und vor allem seine Triterpenoide in den Fokus der aktuellen Forschung [11]. Computergestützte Ergebnisse legen nahe, dass Glycyrrhizinsäure die Anheftung von SARS-CoV-2 erschwert und Glasperin A dessen Replikation in der Wirtszelle stört [12].

Darüber hinaus besitzt Süßholz einen modulierenden Effekt auf das Immunsystem, indem es die Wirksamkeit körpereigenen Cortisols verstärkt, was sich bei COVID19-Erkrankten als hilfreich erwies.

\section{Wirkung auf diverse Körperbereiche}

\section{Atmungstrakt}

Die im Süßholz enthaltenen Saponine, v. a. das Glycyrrhizin, reizen bei oraler Aufnahme den Vagus. Dies regt über einen gastropulmonalen Reflex die Bronchialschleimhaut zur Sekretion eines dünnflüssigen Schleimes an. Dieser verbessert die mukoziliäre Reinigung [5]. Glycyrrhizin, Liquiritigenin und Glycyrrhizinsäure verbesserten in Mäuseversuchen IgE-mediierte Allergien, wie Asthma und Dermatitis [4].

Eine klinische Studie bei Patienten, die zur Lungenoperation einen sehr großlumigen Tracheotubus benötigten, zeigte eindrucksvoll die entzündungshemmenden Süß- 


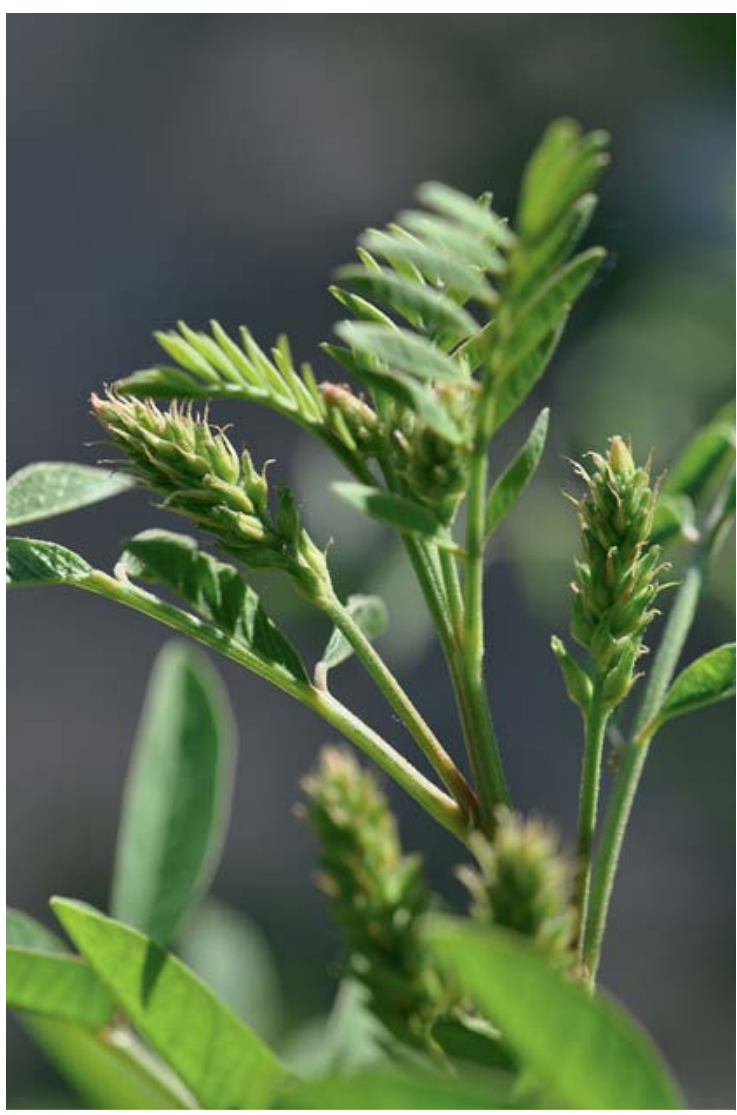

- Abb. 2 Eine Stammpflanze der Süßholzwurzel ist das Echte Süßholz (Glycyrrhiza glabra). Quelle: Sabine Israel

holzwirkungen im oberen Atmungstrakt: Die Patienten gurgelten 5 min vor Anästhesiebeginn 1 min mit wässrigem Süßholzextrakt und zeigten postoperativ deutlich weniger Halsschmerzen, Heiserkeit und Hustenreiz und dadurch einen signifikant besseren Heilungsverlauf. In einem Versuch mit Meerschweinchen hemmten die Polysaccharide aus G. glabra ( $\triangleright$ Abb. 2) den Hustenreiz um $81 \%$ im Vergleich zur Kontrollgruppe [4].

\section{Mundhöhle}

In einer klinischen Studie bei Patienten mit rekurrierender aphtöser Stomatitis zeigte die Süßholzwurzel entzündungshemmende Qualitäten und positive Effekte auf die orale Mundflora. Schmerz, Rötung und die Ausdehnung der nekrotischen Bereiche waren deutlich vermindert [4]. In vitro hemmen verschiedene Süßholzextrakte die Vermehrung oraler Bakterien ohne Resistenzbildung [10]. Die Inhaltsstoffe Licochalcone A und Glabridin besitzen therapeutisches Potenzial bei oralen Candida-albicansInfektionen. Lakritzlipopolysaccharide hemmen als einziger spezifischer Inhibitor die Adhäsion von Porphyromonas gingivalis [7].

\section{Magen-Darm-Trakt}

Süßholz ist für seinen positiven Einfluss auf Magen- und Zwölffingerdarmgeschwüre bekannt. Glycyrrhizin erhöht den Serotonin- und Prostaglandin-Gehalt im MagenDarm-Trakt. Dies steigert die Mukussekretion, verlängert die Überlebenszeit der Magenepithelzellen und reduziert die Pepsinsekretion [7]. Zusätzlich besitzt Glycyrrhizin eine bakteriostatische Wirkung auf Helicobacter pylori, auch auf resistente Stämme. Hydroalkoholischer Süßholzextrakt erzeugt deutliche Spasmolyse am Meerschweinchendarm [4].

Leber

Süßholzextrakt schützt die Leber vor Toxinschäden [7], senkt bei nichtalkoholischer Fettleber die Leberwerte im Serum [4] und hemmt die Bildung von Lebergranulomen [13]. In Japan erhalten Patienten mit chronischer Hepatitis C Glycyrrhizin als Langzeitgabe intravenös. Retrospektive Studien über Jahrzehnte zeigen durch die Behandlung reduzierte Serumaminotransferasen, eine verbesserte Leberhistologie und eine geringere Leberkarzinombildung $[7,11]$.

\section{Haut}

Zahlreiche Tierversuche bestätigen die antiphlogistische Wirkung von Süßholzextrakten auf der Haut. Nicht zuletzt aufgrund corticomimetischer Eigenschaften eignen sie sich zur Behandlung von Ekzemen, Pruritus und atopischer Dermatitis. Hinzu kommt ein Schutz vor UVLicht, aber auch vor anderen Noxen durch das antioxidative Potenzial des Süßholzes [4]. Mehrere Süßholzflavonoide hemmen das Enzym Tyrosinase, welches für die Melaninbildung benötigt wird. Diese depigmentierenden Eigenschaften können allein oder in Kombination mit Carbondioxidlaser zur Behandlung benigner Hyperpigmentationen verwendet werden. Der hydroalkoholische Süßholzextrakt fördert das Haarwachstum [7].

\section{ZNS}

Bei Mäusen zeigt die Gabe eines wässrigen Süßholzextraktes eine deutliche Verbesserung des Lern- und Erinnerungsvermögens. Außerdem wirkt der Süßholzextrakt bei Mäusen dosisabhängig anxiolytisch, sedativ und über Veränderung des Dopaminspiegels auch antidepressiv [7].

Im Rahmen der Alzheimerforschung zeigte Süßholzextrakt neben antioxidativen und antiinflammatorischen Eigenschaften eine weitere neuroprotektive Wirkung: An Neuronen konnte die hemmende Wirkung von Süßholzextrakt auf Fehlfaltungen des Tau-Proteins gezeigt werden [4].

\section{Sonstige Wirkungen}

Es ist nicht möglich, die vielen verschiedenen Studienergebnisse im Rahmen dieses Artikels vorzustellen. Daher möchte ich nur noch einzelne hier hervorheben: 
- Süßholz vermag Magen-, Lungen- und Brustkrebszellen zu hemmen, die Apoptose von Tumorzellen zu fördern [7] und eine Metastasenbildung zu verlangsamen.

- Geröstete Süßholzwurzel unterstützt den Knochenerhalt bei Osteoporose bzw. Parodontose [4].

- Eine Mischung von G. glabra und Boswellia serrata zu gleichen Teilen zeigt gute synergistische Wirkung bei arthritischen Ratten [7].

- Als erster pflanzlicher Thrombininhibitor vermag Glycyrrhizin bei Ratten dosisabhängig die Thrombengröße bis $90 \%$ zu reduzieren [7].

- Süßholzextrakt verbessert die Neovaskularisation der Cornea infolge einer Augenentzündung fast gleichwertig zu Dexametason [9].

- Durch seine östrogene Wirkung vermag Süßholz u.a. Einfluss auf die Prolaktinsynthese und -sekretion zu nehmen. Der Vergleich der östrogenen Wirksamkeit zeigt G. uralensis > G. inflata > G. glabra [4].

\section{Nebenwirkungen}

Glycyrrhizin, das auch dem enterohepatischen Kreislauf unterliegt, verlangsamt in allen Dosierungen mittels Hemmung zweier Enzyme den körpereigenen Cortisolabbau. In sehr hohen Dosen wirkt es auch direkt am Mineralocorticoidrezeptor. Dies führt bei Menschen unter Langzeitgabe zur Ausbildung eines Pseudohyperaldosteronismus mit Ödembildung, Bluthochdruck und Kaliumverlusten [10]. Bei Tieren wurde dies bisher noch nicht beschrieben [14].

\section{Fazit}

Die Süßholzwurzel ist eine klassische Multi-Target-Droge. Sie enthält zahlreiche therapeutisch interessante Inhaltsstoffe und interagiert dementsprechend mit einer Vielzahl von Strukturen im tierischen Organismus. Dies belegt ein breites Spektrum aktueller In-vivo- sowie In-vitro-Studien und spiegelt sich in der breiten Palette von Anwendungen in der Volksmedizin.

In der traditionellen europäischen Medizin wie in der traditionellen chinesischen Medizin schätzt man die Süßholzwurzel seit langem als probates Mittel zur allgemeinen Stärkung und Tonisierung.

Wie wichtig solche Eigenschaften sein können, zeigt die derzeitige COVID-19-Pandemie. Die Süßholzwurzel hat das Potenzial, Virusanheftung und -vermehrung zu unterdrücken, und stärkt gleichzeitig die spezifische und unspezifische Immunantwort. Sie schützt durch ihre antioxidativen Fähigkeiten die Organe - insbesondere Lunge und Blutgefäße - und wirkt über eine Verstärkung der körpereigenen Cortisonwirkung antiinflammatorisch. Zeitgleich könnten Sekundärinfektionen gehemmt und im Krankheitsgeschehen gebildete Toxine besser ausgeschieden werden. Angstlösende und antidepressive Wir- kungen sind zudem eine hilfreiche Unterstützung der Psyche (und damit indirekt des Immunsystems).

Wahrscheinlich ließe sich der therapeutische Nutzen der Süßholzwurzel durch Kombinationen mit weiteren Heilpflanzen oder Pharmaka noch erweitern und durch die hierbei zu erwartenden Synergien noch verstärken.

In China wurde 2003 bei SARS-Patienten begleitend zur Schulmedizin erfolgreich die TCM eingesetzt. Hierbei hat auch die Süßholzwurzel eine wichtige Rolle gespielt [12, 13]. Von derartigen klinischen Erfahrungen könnte man in der derzeitigen Situation profitieren. Mehr Offenheit in Europa gegenüber der phytotherapeutischen Erfahrungsmedizin aus Ost und West wäre sicher eine gute Unterstützung in der Behandlung von Mensch und Tier und nicht zuletzt eine gute Gelegenheit, sich eine eigene Meinung zu bilden.

\section{ZUSAMMENFASSUNG}

Die Süßholzwurzel (Glycyrrhiza radix) wird seit Jahrtausenden aufgrund ihrer antioxidativen, entzündungshemmenden, krampflösenden, sekretolytischen und schleimhautschützenden Eigenschaften geschätzt. Die Pflanze, ihre wichtigsten Inhaltsstoffe und deren unterschiedliche Wirkungen werden kurz beschrieben. Wissenschaftliche Studien untermauern dies und decken zahlreiche weitere Vorzüge dieser Heilpflanze auf. Insbesondere das antivirale Potenzial ist Gegenstand aktueller Forschung.

\section{SUMMARY}

\section{Licorice root (Glycyrrhiza radix)}

Licorice root (Glycyrrhiza radix) is used and valued since millennia for its antioxidant, antiphlogistic, antispasmodic and secretolytic effects as well as its protective properties of the mucous membrane. A concise plant description and its most important ingredients as well as their various effects are listed. Scientific studies underpin these and show numerous advantages like the immune-modulating qualities of this medicinal plant. Especially the antiviral potential is of great importance in the latest research.

\section{Autorin}




\section{Korrespondenzadresse}

Sabine Israel

Philipp-Försch-Str. 12

55257 Budenheim

Deutschland

sa.israel@gmx.de

\section{Literatur}

[1] Suwanda S, Tian L. Chinesische Arzneimitteltherapie. 1. Auflage. Stuttgart: Hippokrates; 2005

[2] Putscher M. Das Süssholz und seine Geschichte [Dissertation]. Institut für Geschichte der Universität Köln, 1965

[3] Blaschek W. Wichtl-Teedrogen und Phytopharmaka. 6. Auflage. Stuttgart: Wissenschaftliche Verlagsgesellschaft Stuttgart; 2016

[4] Hosseinzadeh H, Nassiri-Asl M. Pharmacological Effects of Glycyrrhiza spp. and its Bioactive Constituents: Update and Review. Phytother. Res. 2015; 29: 1868-1886

[5] Bühring U. Praxis-Lehrbuch der modernen Heilpflanzenkunde. 1. Auflage. Stuttgart: Sonntag; 2005

[6] Mayer M, Zbinden M, Vogl CR et al. Forsch Komplementmed 2014; 21: 375-386

[7] Pastorino G, Cornara L, Soares S et al. Liqourice (Glycyrrhiza glabra): A phytochemical and pharmacological review. Phytother.Res. 2018; 32:2323-2339

[8] Alagawany M, Elnesr S, Farag M et al. Use of Licorice (Glycyrrhiza glabra) Herb as a Feed Additive in Poultry: Current Knowledge and Prospects. Animals (Basel) 2019; 9(8): 536

[9] Schmidt S, Heimesaat M, Fischer $A$ et al. Saponins increase susceptibility of vancomycin-resistant enterococci to antibiotic compounds. Eur J Microbiol Immunol (BP)2014; 4: 204-212
[10] Batiha G, Beshbishy A, El-Mleeh A et al. Traditional Uses, Bioactive Chemical Constituents, and Pharmacological and Toxicological Activities of Glycyrrhiza glabra L. (Fabaceae). Biomolecules 2020; 10: 352

[11] Fiore C, Eisenhut M, Krausse R et al. Antiviral Effects of Glycyrrhiza species Phytother. Res. 2008; 22:141-148

[12] Sinha S, Prasad S, Islam M et al. Identification of bioactive compounds from Glycyrrhiza glabra as possible inhibitor of SARS-CoV-2 spike glycoprotein and non-structural protein15: a pharmacoinformatics study. Journal of Biomolecular Structure and Dynamics https://doi.org./doi:10.1080/ 07391102.2020 .1779132

[13] Jia W, Gao W. Is Traditional Chinese Medicine Useful in the Treatment of SARS? Phytother. Res. 2003; 17: 840-841

[14] Brendieck-Worm C, Melzig M. Phytotherapie in der Tiermedizin. 1. Auflage. Stuttgart: Thieme; 2018

[15] Shah S, Wahid F, Khan N et al. Inhibitory Effects of Glycyrrhiza glabra and Ist Major Constituent Glycyrrhizin on InflammatioAssoziated Corneal Neovascularisation. doi:10.1155/2018/ 8438101

[16] Wang L,Yang R, Yuan B et al. Tha antiviral and antimicrobial activities of licorice, a widely-used Chinese herb. Acta Pharmaceutica Sinica B 2015; 5: 310-315

\section{Bibliografie}

Zeitschrift für Ganzheitliche Tiermedizin 2020; 34: 140-146 DOI 10.1055/a-1258-8015

ISSN $\quad 0939-7868$

(c) 2020. Thieme. All rights reserved.

Georg Thieme Verlag KG, Rüdigerstraße 14,

70469 Stuttgart, Germany 